\title{
The Usefulness of Nasal Packing with Vaseline Gauze and Airway Silicone Splint after Closed Reduction of Nasal Bone Fracture
}

\author{
Hyo Young Kim, Sin Rak Kim, Jin Hyung Park, Yea Sik Han \\ Department of Plastic Surgery, Kosin University Gospel Hospital, Kosin University School of Medicine, Busan, Korea
}

Background Packing after closed reduction of a nasal bone fracture causes inconvenient nasal obstruction in patients. We packed the superior meatus with Vaseline gauze to support the nasal bone, and packed the middle nasal meatus with a Doyle Combo Splint consisting of an airway tube, a silastic sheet, and an expandable sponge to reduce the inconvenience. In addition, we aimed to objectively identify whether this method not only enables nasal respiration but also sufficiently supports the reduced nasal bone.

Methods Nasal ventilation was measured via spirometry 1 day before surgery and compared to 1 day after surgery. To compare support of the reduced nasal bone by the 2 methods, 2 plastic surgeons assessed the displacementon $X$-rays taken after the surgery and after removing the packing. The extent of nasal obstruction, dry mouth, sleep disturbance, headache, and swallowing difficulty were compared with visual analog scales (VAS) on a pre-discharge survey. Results In the experimental group, the nasal respiration volume 1 day after surgery remained at $71.3 \% \pm 6.84 \%$ on average compared to 1 day prior to surgery. Support of the reduced bone in the experimental group $(2.80 \pm 0.4)$ was not significantly different from the control group ( $2.88 \pm 0.33$ ). The VAS scores for all survey items were lower in the experimental group than in the control group, where a lower score indicated a lower level of inconvenience.

Conclusions The nasal cavity packing described here maintained objective measures of nasal respiration and supported the reduced bone similar to conventional methods. Maintaining nasal respiration reduced the inconvenience to patients, which demonstrates that this packing method is useful.

Keywords Nasal surgical procedures / Nasal bone / Airway management

\author{
Correspondence: Yea Sik Han \\ Department of Plastic Surgery, Kosin \\ University Gospel Hospital, Kosin \\ University School of Medicine, 262 \\ Gamcheon-ro, Seo-gu, Busan \\ 602-702, Korea \\ Tel: +82-51-990-6131 \\ Fax: +82-51-990-3034 \\ E-mail: hanplastic1@naver.com
}

This article was presented at the 69th Congress of the Korean Society of Plastic and Reconstructive Surgeons on November 11-13, 2011, in Seoul, Korea.

No potential conflict of interest relevant to this article was reported.

Received: 2 Jul 2012 • Revised: 7 Aug 2012 • Accepted: 22 Aug 2012

pISSN: 2234-6163・ elSSN: 2234-6171 • http://dx.doi.org/10.5999/aps.2012.39.6.612 • Arch Plast Surg 2012;39:612-617

\section{INTRODUCTION}

The packing method using paraffin mesh or Vaseline gauze after the reduction of a nasal bone $[1,2]$ can trigger symptoms such as nasal obstruction, mucosal adhesion, sleep disturbance, headache, mouth dryness, and dysphagia. Nasal obstruction can give rise to especially severe discomfort in patients after surgery. Therefore, the ideal nasal packing method should include sufficient splinter support and result in less discomfort associated with nasal obstruction.

To reduce patient discomfort, studies have assessed the performance of packing methods that use an airway tube Merocel $[3,4]$, a rolled silastic sheet [5], or a bronchodilator [6]. These studies, however, only evaluated results through patient surveys, 
lacking objective measures of nasal respiration. Furthermore, there is a dearth of studies comparing how well new and conventional methods support the reduced bone.

We developed a nasal bone support using Vaseline gauze in the superior meatus, and a Doyle Combo Splint (DCS, Boston Medical Products Inc., Westborough, MA, USA) (Fig. 1) combined with an airway tube, a silastic sheet, and Rhinocell (expandable sponge) in the middle meatus. The specific aims of this article were as follows: 1) Determine whether this method maintains nasal respiration, and determine whether the nasalrespiration volume can be measured quantitatively and objectively. 2) Determine whether support of the reduced bone is sufficient and similar to the conventional method. 3) Compare subjective patient discomfort to conventional Vaseline gauze packing.

\section{METHODS}

\section{Patients}

This trial was conducted on 120 male patients between 20 and
50 years old who underwent closed reduction of a nasal fracture from December 2008 to October 2010. Sixty patients were randomized into a control group who had nasal packing using Vaseline gauze alone, and the remaining 60 patients (experimental group) had nasal packing with Vaseline gauze and a DCS. The mean age of the control group and experimental group were $38.5 \pm 11.3$ years and $37.3 \pm 11.5$ years, respectively. Patients under 20 years and over 50 years of age, as well as all female patients, were excluded due to the likelihood of a distinct nasal respiration volume. Fracture sites were identified in all patients through preoperative radiography and computed tomography (CT) scans.

\section{Surgical technique}

After reducing the nasal fractures and supporting the superior meatus with Vaseline gauze, ointment was applied to the DCS, which was inserted into the middle meatus of both nasal cavities (Fig. 2). If nasal respiration decreased due to hematoma or intranasal discharge, the airway tube was sucked by applying negative pressure with a 10-Fr Nelaton tube. Ventilation was
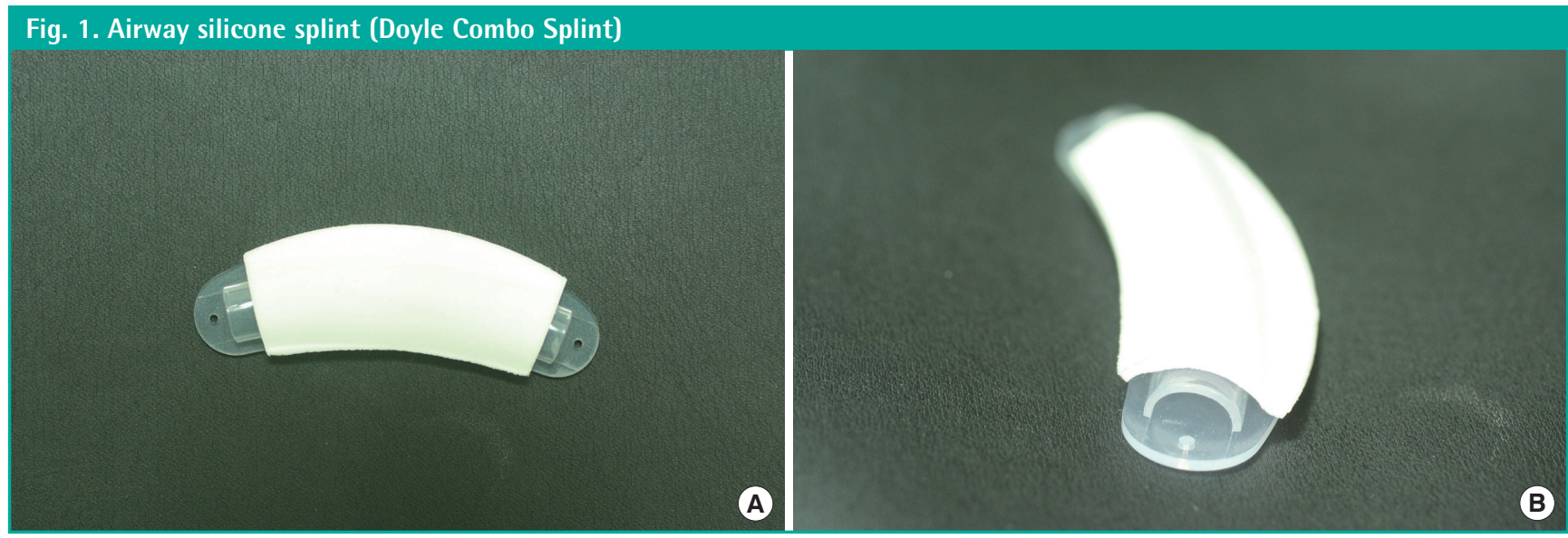

Fig. 2. Illustration of nasal packing with an airway silicone splint and Vaseline gauze

(A) Status of packing in the sagittal view. (B) Frontal view.

(A)

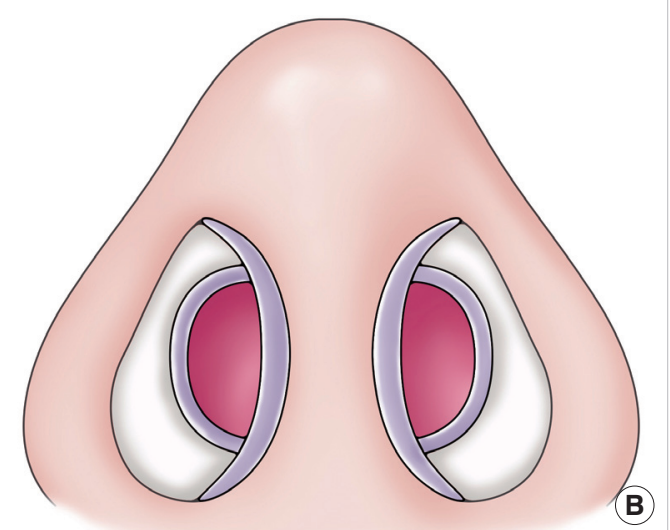


measured using an airway tube and through plain radiography on the day of the surgery (Fig. 3).

\section{Nasal respiration comparison}

To objectively compare ventilation through the airway tube, the natural nasal expiratory volume was measured using spirometry 1 day before and after surgery in all of the patients. The accumulated nasal respiration was determined by measuring the natural nasal expiratory volume for 6 seconds with a closed mouth. Air leakage was prevented by closing the nose and mouth with an oxygen mask and by connectting an oxygen mask to a spirometry tube with transparent film.

\section{Reduced-support comparison}

To compare support of the reduced bone of each group, plain $\mathrm{X}$-ray images (Waters view, both lateral views) were taken right after surgery and immediately after removing the packing. Two blinded plastic surgeons compared X-rays and scored the support on a scale of 1 to 3 . The final score was derived based on a consensus of the 2 surgeons (Table 1). A displacement was defined as a depression or elevation in the bone fragment on the

\section{Table 1. Displacement scoring system}

\begin{tabular}{|ll|}
\hline Score & \multicolumn{1}{c|}{ Description } \\
\hline 3 & No displacement or bony gap widening was observed. \\
2 & No displacement was observed, but bony gap widening was observed. \\
1 & Both displacement and bony gap widening were observed. \\
\hline
\end{tabular}

X-ray image taken after removing the packing. Gaps between the bone fragments that widened without displacement were evaluated as bony gap widening.

\section{Discomfort due to nasal packing}

All patients completed a survey of nasal obstruction, dry mouth, sleep disturbance, headache, and swallowing difficulty immediately before removing the packing, and results were assessed with visual analog scales (VAS) scores.

\section{Statistical analysis}

For statistical analysis, SPSS ver.18.0 (IBM, Armonk, NY, USA) was used. Measured values are expressed as averages and standard deviations. The experimental and control groups were compared through the Student's t-test and were considered significant when P-value was $<0.05$.

\section{RESULTS}

\section{Measurement of the nasal respiration via spirometry}

Packing accidentally fell out in 5 of the control patients, who were excluded from the analyses. The remaining 55 patients had a nasal respiration of $3.08 \pm 0.17 \mathrm{~L}$ before surgery and $0 \mathrm{~L} 1$ day after surgery. Six patients in the experimental group had no nasal respiration, as measured via spirometry 1 day after surgery, and were excluded (see "DISCUSSION" section). The remaining 54 had nasal respiration of $3.05 \pm 0.15 \mathrm{~L}$ before surgery and $1.82 \pm 0.25 \mathrm{~L} 1$ day after surgery. Nasal respiration after surgery

Fig. 3. Plain films 1 day after surgery

Ventilation was achieved through an airway tube. (A) Waters view. (B) Lateral view of the nasal bone.
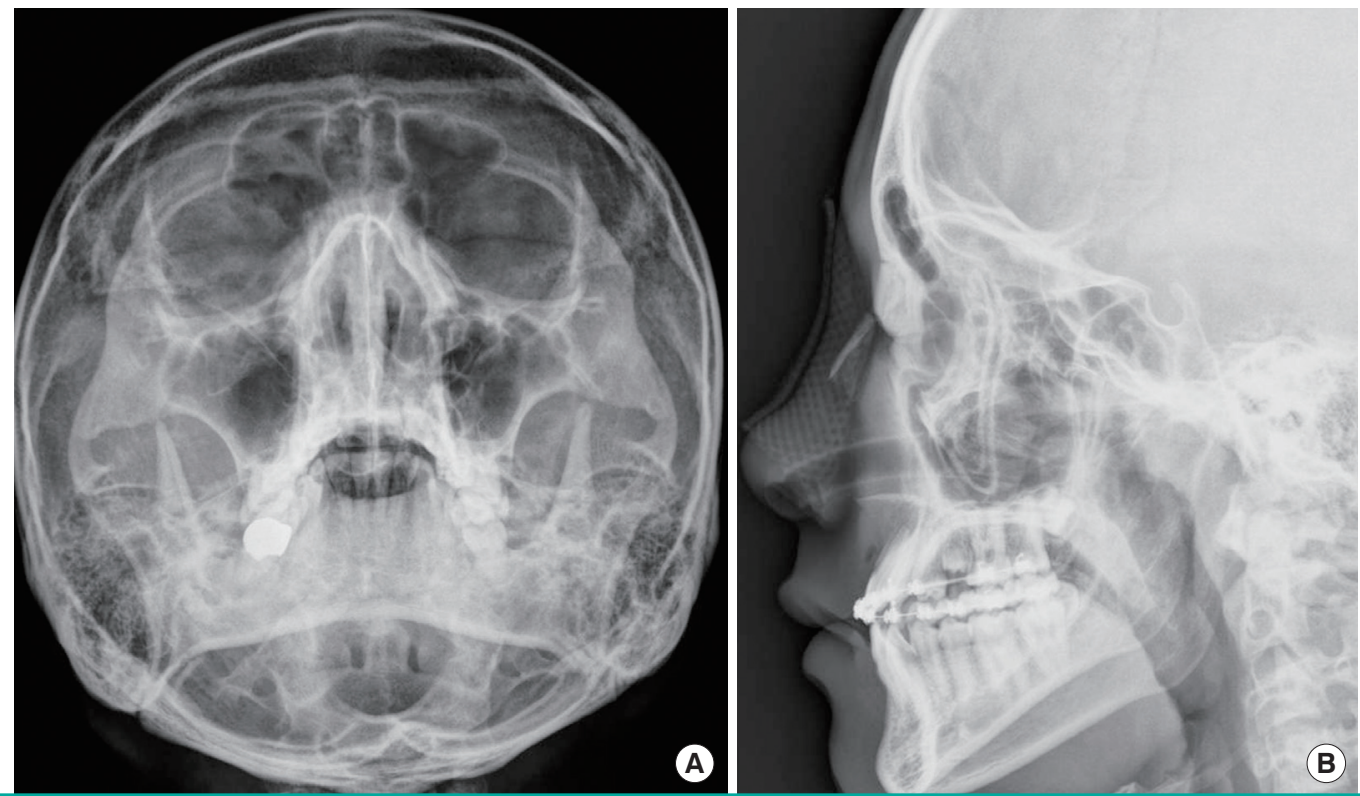
was an average of $71.3 \% \pm 6.84 \%$ of that before surgery. Nasal respiration after surgery was significantly different between the two groups $(\mathrm{P}<0.001)$ (Fig. 4).

\section{Comparison of support for reduced bones}

The average support scores were $2.80 \pm 0.4$ in the experimental group and $2.88 \pm 0.33$ in the control group, which are not significantly different $(\mathrm{P}=0.052)$.

\section{Discomfort due to nasal packing}

The VAS scores of nasal obstruction, dry mouth, sleep disturbance, headache, and swallowing difficulty were $44.6 \pm 15.1$, $36.4 \pm 17.2,40.8 \pm 16.2,65.2 \pm 15.2$, and $32.3 \pm 20.8$, respectively, in the experimental group, excluding the 6 patients without nasal ventilation. The VAS scores were $79.6 \pm 10.7,67.8 \pm 12$, $68.9 \pm 17.4,69.6 \pm 16.2$, and $64.6 \pm 15.5$, respectively, in the control group, excluding the 5 patients with accidental packing removal (Fig. 5). The experimental group had significantly lower scores than the control group for all items except headache $(\mathrm{P}<0.001)$. Although the headache score was lower in the experimental group, the 2 groups were not significantly dif-

Fig. 4. Comparison of the accumulated nasal respiration 1 day before surgery and 1 day after surgery

The nasal respiration volume after surgery remained at $72 \%$ of baseline when a complex ventilation splint was used.

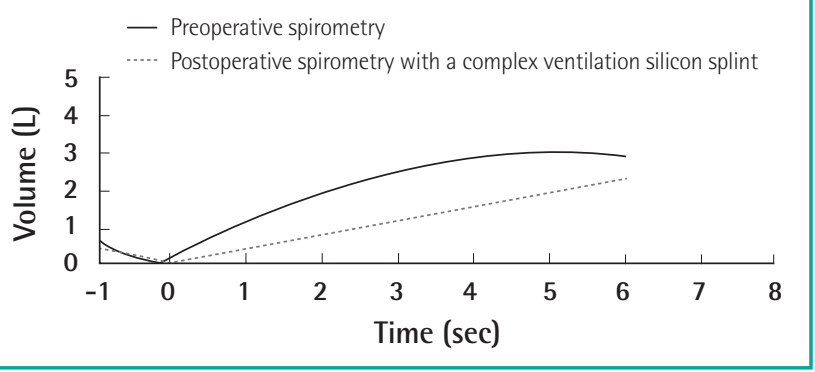

ferent $(\mathrm{P}=0.502)$. No patients in the experimental group had accidental packing removal or filler extrusion to the nostrils or nasopharynx; however, as mentioned earlier, accidental packing removal occurred in a total of 5 patients $(8.3 \%)$ in the control group. The details of the results are given in Table 2.

\section{DISCUSSION}

The existing nasal packing method can cause discomfort, such as nasal obstruction, headache, and dry mouth, and complications, such as nasal septal perforation and toxic shock syndrome [7]. Nasal obstruction not only causes severe discomfort but can also cause sleep apnea, high blood pressure, and nocturnal oxygen desaturation $[8,9]$. Therefore, various methods have been reported for supporting the reduced nasal bone without

Fig. 5. Comparison of VAS scores according to surgical method

The experimental group had significantly less severe discomfort than the control group. VAS, visual analog scales.

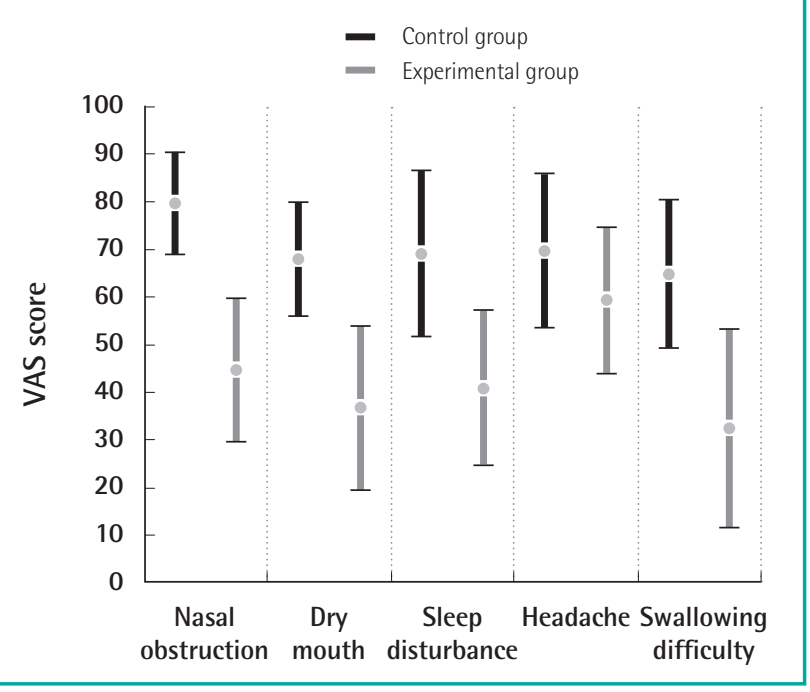

Table 2. Results of preoperative and postoperative nasal respiration, support scores for reduced bone, and VAS scores due to packing

\begin{tabular}{|c|c|c|c|}
\hline Variable & Control group $(n=55)$ & Experimental group $(n=54)$ & P-value \\
\hline Preoperative nasal respiration $(\mathrm{L})$ & $3.08 \pm 0.17$ & $3.05 \pm 0.15$ & 0.104 \\
\hline Postoperative nasal respiration $(\mathrm{L})$ & 0 & $1.82 \pm 0.25$ & $<0.001$ \\
\hline Nasal respiration after surgery: percentage of that before surgery (\%) & 0 & $71.3 \pm 6.84$ & $<0.001$ \\
\hline Support scores for reduced bone & $2.88 \pm 0.33$ & $2.80 \pm 0.4$ & 0.052 \\
\hline \multicolumn{4}{|l|}{ Discomfort due to packing } \\
\hline Nasal obstruction & $79.6 \pm 10.7$ & $44.6 \pm 15.1$ & $<0.001$ \\
\hline Dry mouth & $67.8 \pm 12$ & $36.4 \pm 17.2$ & $<0.001$ \\
\hline Sleep disturbance & $68.9 \pm 17.4$ & $40.8 \pm 16.2$ & $<0.001$ \\
\hline Headache & $69.6 \pm 16.2$ & $65.2 \pm 15.2$ & 0.502 \\
\hline Swallowing difficulty & $64.6 \pm 15.5$ & $32.3 \pm 20.8$ & $<0.001$ \\
\hline
\end{tabular}


the standard packing $[10,11]$. In clinical practice, however, packing is used extensively to support reduced bone.

We devised a method to minimize discomfort by inserting DCS into the middle meatus to allow ventilation while supporting the reduced bone with Vaseline gauze. DCS, a complex airway silicone splint, is made of a $7 \times 2 \mathrm{~cm}$ silicone sheet to support the nasal septum and a $0.8 \mathrm{~cm}$ diameter semicircular ventilating tube, and is surrounded by Rhinocell. The silicone sheet and ventilating tube enables smooth nasal respiration by filling the overall meatus.

Spirometry was used to quantify the nasal respiration through the ventilating tube. The natural expiratory flow is associated with the extent of patient discomfort, and was measured as the accumulated natural nasal expiration volume via unforced expiration for 6 seconds.

The accumulated expiratory flow in the experimental group after surgery remained at $71.3 \% \pm 6.84 \%$ of that 1 day before surgery, excluding 6 patients who did not have expiratory flow. Before surgery, most patients had a great deal of expiratory flow at the initial stage, which flattened off. After surgery, the expiratory flow was consistent during the early and later stages. This difference is likely due to obstruction because expiration is carried out only through the ventilating tube. The relatively smooth ventilation, however, amounted to $71.3 \% \pm 6.84 \%$ of that before surgery. A broader ventilating pipe could have improved these results to some extent, but the support and ease of use must be considered.

In the survey of patient discomfort, the experimental group had significantly lower VAS scores for nasal obstruction, dry mouth, sleep disturbance, headache, and swallowing than the control group. Negative pressure was prevented from building up in the nasopharynx and middle ear by enabling early nasal respiration, thus reducing uncomfortable symptoms [12]. In addition, because nasal respiration was possible, there was no need for oral respiration, which improved symptoms, such as dry mouth and swallowing difficulty. While the experimental group had lower headache scores, the difference was not statistically significant. Pressure in the sinuses when Vaseline gauze is used to pack the superior meatus can result in painful headaches. The displacement of the reduced bone between reduction and the time of packing removal in the experimental and control groups was not significantly different. Some suggest that sufficient support of reduced bone can only be achieved by packing the superior meatus and ethmoidal crest with Vaseline gauze. In the conventional method, the Vaseline gauze comes out of the nasal cavity or goes down to the nasopharynx. In some cases, patients remove the gauze due to discomfort, resulting in a lack of appropriate support for a sufficient time. In this study, 8.3\% of the control group, but no one in the experimental group, removed the gauze. DCS packing of the middle meatus provides sufficient support, which prevents the Vaseline gauze from extruding.

Another advantage of the proposed method is that it has less pain and bleeding upon packing removal, and can be useful in providing support after reducing septal fractures. Merocel, which has been extensively used as a packing material of late, causes excessive pain and bleeding upon removal compared to Vaseline gauze because it tends to adhere to the mucosa $[13,14]$. Furthermore, Merocel is less flexible than Vaseline gauze, and it is difficult to accurately insert into the superior meatus due to its shape. The lateral side of DCS consists of Rhinocell (expandable sponge). Rhinocell, composed of polyvinyl, provides a smooth surface to minimize tissue in growth. In addition, its high tensile strength prevents shedding or tearing the mucosa. Rhinocell is expanded by normal saline, and delivers appropriate pressure to the nasal mucosa and septum. Thus, it has less risk of moving the DCS and provides support to the reduced nasal septum. The medial side of DCS stably maintains the corrected nasal septum along with Rhinocell because it is made of a silicone sheet. Furthermore, if damage occurs to the mucous membrane during surgery, it maintains moisture by covering the damaged site, and promotes quick healing by preventing additional mucosal damage and irritation.

There are some precautions, however, to using this method. In patients with a narrow nasal cavity, including children under 10 years old, it can be difficult to insert a splint. A portion of the silicone sheet and expandable sponge can be cut off, however, to reduce the size outside the ventilating tube.

Six patients had no nasal respiration volume in the spirometry despite having an airway silicone splint, but still recorded high VAS scores (data not shown). In these patients, there was likely severe nasal bleeding during reduction of the nasal bone, or the Vaseline gauze blocked the end of the ventilating opening. Their airway was not opened because the ventilating opening was blocked due to clotted blood or continuous discharge. Therefore, it is ideal to check whether the ventilating tube can be penetrated by a thin suction tip so that the airway can be appropriately maintained. On the day of the surgery, frequently checking nasal respiration and opening the ventilating tube by suction with a thin tube if blocked due to blood clotting or secretion will help ease discomfort.

A limitation of our study is that CT scanning was not used to compare the support of the reduced bone between groups. Although CT provides a more accurate evaluation, its use can be cost-ineffective. Several studies conducted closed reduction with a $\mathrm{C}$-arm, achieving accurate results by observing the bone with continuous imaging. The results were confirmed by CT 
scanning $[15,16]$. Therefore, it is concluded that relatively suitable assessments can be obtained using plain film.

In conclusion, the authors were able to pack the superior meatus with Vaseline gauze after closed reduction, and maintained nasal ventilation of the middle meatus by filling it with DCS. This was quantitatively determined through spirometry. In addition, the decrease in uncomfortable symptoms was confirmed through a survey. The suitable support of this packing method was determined by X-ray. We conclude that this method of nasal packing is useful in reducing discomfort and providing appropriate support.

\section{REFERENCES}

1. Huizing E, Pirsig W, Wentges R, et al. Unanimity and diversity in nasal surgery. Rhinol Suppl 1989;9:15-23.

2. Schultz LW. Nasal splints: advantages, disadvantages and author's modifications. Am J Surg 1957;94:897-9.

3. Rhee SC, Kim JS. A simple method of fabricating nasal packing armed with ventilation tube. J Craniofac Surg 2008;19: 1385-6.

4. Jung YJ, Choi YW, Nam SH, et al. The usefulness of airway tube $\operatorname{Merocel}(\mathrm{R})$ on treatment of nasal bone fracture. J Korean Cleft Palate-Craniofac Assoc 2009; 10:14-8.

5. Son KM, Yang JY, Kim GB, et al. The effect of nasal packing with rolled silastic sheet after closed reduction of nasal bone fracture. J Korean Soc Plast Reconstr Surg 2011;38:602-8.

6. Kim JY, Kim YH. The usefulness of a bronchodilator after the closed reduction of nasal bone fracture. J Korean Cleft
Palate-Craniofac Assoc 2011;12:22-7.

7. Fairbanks DN. Complications of nasal packing. Otolaryngol Head Neck Surg 1986;94:412-5.

8. Ogretmenoglu O, Yilmaz T, Rahimi K, et al. The effect on arterial blood gases and heart rate of bilateral nasal packing. Eur Arch Otorhinolaryngol 2002;259:63-6.

9. Johannessen N, Jensen PF, Kristensen S, et al. Nasal packing and nocturnal oxygen desaturation. Acta Otolaryngol Suppl 1992;492:6-8.

10. Park S, Iwasaki Y. Intranasal Kirschner wire splinting of nasal fractures. Plast Reconstr Surg 2000;106:952-3.

11. Burm JS, Oh SJ. Indirect open reduction through intercartilaginous incision and intranasal Kirschner wire splinting of comminuted nasal fractures. Plast Reconstr Surg 1998; 102:342-9.

12. Garth RJ, Brightwell AP. A comparison of packing materials used in nasal surgery. J Laryngol Otol 1994;108:564-6.

13. Ruddy J, Brain D, Sudesh RR, et al. A prospective trial of Merocel packs. Rhinology 1991;29:281-5.

14. Acioglu E, Edizer DT, Yigit O, et al. Nasal septal packing: which one? Eur Arch Otorhinolaryngol 2012;269:1777-81.

15. Kang SJ, Kim JW. Proposed method for closed reduction of impacted nasal bone fractures using a Kirschner wire and a C-arm. J Oral Maxillofac Surg 2012;70:1393-7.

16. Han DS, Han YS, ParkJH. A new approach to the treatment of nasal bone fracture: the clinical usefulness of closed reduction using a C-arm. J Plast Reconstr Aesthet Surg 2011; 64:937-43. 


\section{Discussion}

\section{Postoperative Evaluation of Silicone Airway Splint in Nasal Bone Fracture Treatment}

Yong-Ha Kim

Department of Plastic and Reconstructive Surgery, Yeungnam University Medical Center, Yeungnam University College of Medicine, Daegu, Korea

This is a quantitative and objective study using spirometry to verify that the airway is maintained in the case of intranasal packing with a Doyle Combo Splint (Boston Medical Products, Westborough, MA, USA) [1]. The patient's discomfort during the nasal packing was scored on a survey using a visual analogue scale. Thus, the topic of this study is not a new one in which the airway was maintained after the nasal packing with the Doyle Combo Splint, and patient's discomfort was reduced because nasal respiration was possible. The researchers evaluated the patients using plain X-ray images postoperatively and immediately after removing the nasal packing. They reported that there was no difference in the degree of support for the reduced bone in the control group and experimental group using a Doyle Combo Splint with. Considering the purpose of the nasal packing, this result is the main point of this study.

However, the research method is insufficient for producing objective scientific results. Plain X-ray images can be changed depending on the angle because they are two-dimensional images. Some simple fractures can be displayed well on dedicated $\mathrm{X}$-ray projections. On the other hand, complex fractures can only be partially evaluated because of the overlap of the various structures in the craniofacial skeleton, the complexity of which demands considerable expertise in evaluation [2]. Us- ing computed tomography (CT) is limited due to the cost and radiation exposure; however, studies with $\mathrm{CT}$ are necessary for objective evaluation. Another limitation of this study is that the short-time progress was only observed for the evaluation of the patient's discomfort during the nasal packing.

A significant conclusion could be drawn if patient satisfaction, nasal deformities, and possible complications after removal of the nasal packing had been compared in the control and the experimental groups over an extended period of observation $[3,4]$.

\section{REFERENCES}

1. Jones AS, Viani L, Phillips D, et al. The objective assessment of nasal patency. Clin Otolaryngol Allied Sci 1991;16:206-11.

2. Hardt N, Kuttenberger J. Craniofacial trauma: diagnosis and management. Berlin: Springer-Verlag; 2010.

3. Park WY, Kim YH. A clinical study of the nasal bone fracture according to Stranc classification. J Korean Soc Plast Reconstr Surg 2008;35:289-94.

4. Lee JH, Park WY, Nam HJ, et al. Complications of the nasal bone fractures according to the Stranc classification.J Korean Cleft Palate-Craniofac Assoc 2008;9:62-6.

Correspondence: Yong-Ha Kim

Department of Plastic and Reconstructive Surgery, Yeungnam University Medical Center, Yeungnam University College of Medicine, 170 Hyeonchung-ro, Nam-gu, Daegu 705-717, Korea Tel: +82-53-620-3481, Fax: +82-53-626-0705, E-mail: yhkim@med.yu.ac.kr

No potential conflict of interest relevant to this article was reported.

Received: 28 Oct 2012 • Revised: 31 Oct 2012 • Accepted: 1 Nov 2012

pISSN: 2234-6163・ elSSN: 2234-6171

http://dx.doi.org/10.5999/aps.2012.39.6.618• Arch Plast Surg 2012;39:618

Copyright (C) 2012 The Korean Society of Plastic and Reconstructive Surgeons

This is an Open Access article distributed under the terms of the Creative Commons Attribution Non-Commercial License (http://creativecommons.org/

licenses/by-nc/3.0/) which permits unrestricted non-commercial use, distribution, and reproduction in any medium, provided the original work is properly cited. 